\title{
X-ray spectral evolution of SAX J1747.0-2853 during outburst activity and confirmation of its transient nature
}

\author{
N. Werner ${ }^{1,2}$, J. J. M. in't Zand ${ }^{2,3}$, L. Natalucci ${ }^{4}$, C. B. Markwardt ${ }^{5,6}$, \\ R. Cornelisse ${ }^{2,3,7}$, A. Bazzano ${ }^{4}$, M. Cocchi ${ }^{4}$, J. Heise $^{2,3}$, and P. Ubertini ${ }^{4}$ \\ ${ }^{1}$ Faculty of Sciences, P. J. Safarik University, Moyzesova 16, 04001 Kosice, Slovak Republic \\ 2 SRON National Institute for Space Research, Sorbonnelaan 2, 3584 CA Utrecht, The Netherlands \\ 3 Astronomical Institute, Utrecht University, PO Box 80000, 3508 TA Utrecht, The Netherlands \\ ${ }^{4}$ Istituto di Astrofisica Spaziale (CNR), Area Ricerca Roma Tor Vergata, via del Fosso del Cavaliere, 00133 Roma, Italy \\ 5 NASA Goddard Space Flight Center, Code 662, Greenbelt, MD 20771, USA \\ ${ }^{6}$ Dept. of Astronomy, University of Maryland, College Park, MD 20742, USA \\ 7 Dept. of Physics and Astronomy, University of Southampton, Hampshire SO17 1BJ, UK
}

Received 2 September 2003 / Accepted 29 November 2003

\begin{abstract}
SAX J1747.0-2853 is an X-ray transient which exhibited X-ray outbursts yearly between 1998 and 2001, and most probably also in 1976. The outburst of 2000 was the longest and brightest. We have analyzed X-ray data sets that focus on the 2000 outburst and were obtained with BeppoSAX, XMM-Newton and RXTE. The data cover unabsorbed 2-10 keV fluxes between 0.1 and $5.3 \times 10^{-9} \mathrm{erg} \mathrm{s}^{-1} \mathrm{~cm}^{-2}$. The equivalent luminosity range is $6 \times 10^{35}$ to $2 \times 10^{37} \mathrm{erg} \mathrm{s}^{-1}$. The $0.3-10 \mathrm{keV}$ spectrum is well described by a combination of a multi-temperature disk blackbody, a hot Comptonization component and a narrow Fe-K emission line at 6.5 to $6.8 \mathrm{keV}$ with an equivalent width of up to $285 \mathrm{eV}$. The hydrogen column density in the line of sight is $(8.8 \pm 0.5) \times 10^{22} \mathrm{~cm}^{-2}$. The most conspicuous spectral changes in this model are represented by variations of the temperature and radius of the inner edge of the accretion disk, and a jump of the equivalent width of the Fe-K line in one observation. Furthermore, 45 type-I X-ray bursts were unambiguously detected between 1998 and 2001 which all occurred during or close to outbursts. We derive a distance of $7.5 \pm 1.3 \mathrm{kpc}$ which is consistent with previous determinations. Our failure to detect bursts for prolonged periods outside outbursts provides indirect evidence that the source returns to quiescence between outbursts and is a true transient.
\end{abstract}

Key words. accretion, accretion disks - X-rays: binaries - X-rays: bursts - X-rays: individual: SAX J1747.0-2853

\section{Introduction}

The transient X-ray source SAX J1747.0-2853 was discovered during an observation of the Galactic bulge with the BeppoSAX Wide-Field Cameras (WFCs) in March 1998 (in't Zand et al. 1998). The position of the source is consistent with that of the transient source GX+0.2-0.2 detected by Ariel V in 1976 (Proctor et al. 1976). The source went into outburst again in March 2000 (Markwardt et al. 2000) and September 2001 (Wijnands et al. 2002). Type I X-ray bursts were detected at the discovery proving that SAX J1747.0-2853 is a weakly magnetized neutron star (type I X-ray bursts are thought to be due to thermonuclear flashes on the surface of a neutron star; for reviews see Lewin et al. 1993; Strohmayer $\&$ Bildsten 2003). The distance as determined from equalizing the peak bolometric flux of photospheric radius expansion bursts to the Eddington limit expected for a neutron star was measured by Sidoli et al. (1998) and Natalucci et al. (2000)

Send offprint requests to: $\mathrm{N}$. Werner, e-mail: werner@ta3.sk to be between 8 and $10 \mathrm{kpc}$. Given that the currently best estimate for the distance to the Galactic center is $8.0 \pm 0.4 \mathrm{kpc}$ (Eisenhauer et al. 2003) and that the angular distance of $19^{\prime}$ translates to a linear tangential distance of only $44 \mathrm{pc}$, it is quite possible that the source is located within only $\approx 100$ pc from the Galactic center. According to Sidoli et al. (1998) the severe interstellar absorption in this region hampers the search for the optical counterpart of the source. The apparent $J$ magnitude is predicted to be larger than 30 for the low mass companion.

The source was serendipitously observed with Chandra/ACIS in 2001 July (Wijnands et al. 2002), when it was not in a bright outburst. The $0.5-10 \mathrm{keV}$ unabsorbed $\mathrm{X}$-ray luminosity for a distance of $9 \mathrm{kpc}$ was $3 \times 10^{35} \mathrm{erg} \mathrm{s}^{-1}$. The spectrum was consistent with a power law with a photon index of about 2 while showing no sign of an $\mathrm{Fe}-\mathrm{K}$ emission line. Since the luminosity is at least an order of magnitude larger than expected for quiescence, it appears that the classification of SAX J1747.0-2853 as transient was premature. 


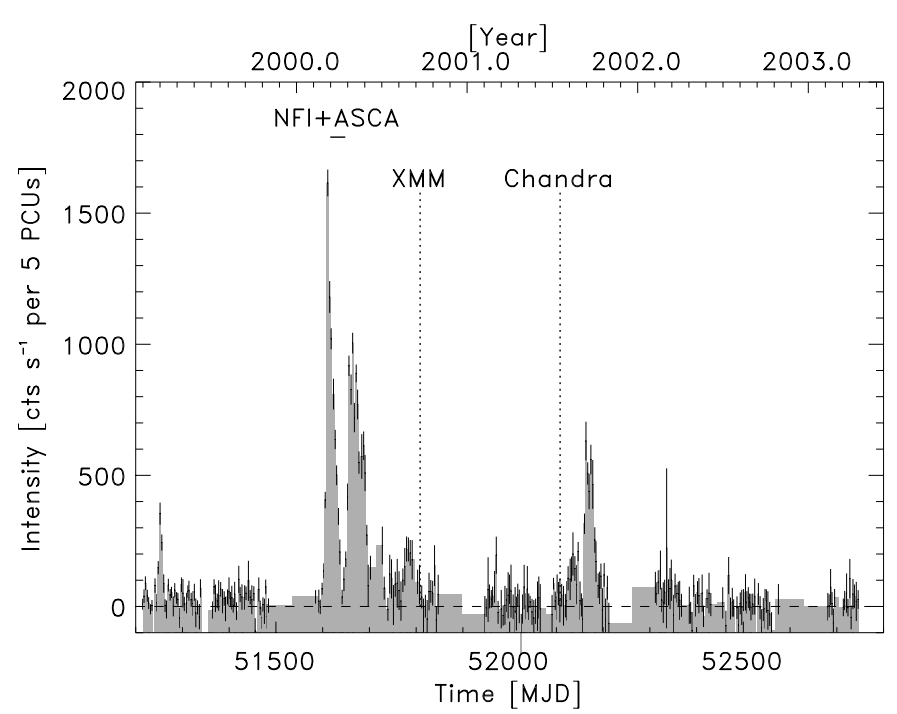

Fig. 1. The PCA lightcurve of the SAX J1747.0-2853 obtained between 1999 February 6 and 2003 March 30. The times of the five BeppoSAX NFI observations are indicated by the horizontal line and the observations with XMM-Newton and Chandra are indicated by the vertical dashed lines.

The possibility of the implied low accretion levels makes it a particularly interesting object for further study.

In this light we have analyzed X-ray data of SAX J1747.0-2853 that were obtained in 2000 when the source was fairly active. The data were collected with the Narrow-Field Instruments (NFIs) and Wide Field Cameras (WFCs) on BeppoSAX, the European Photon Imaging Camera (EPIC) PN and MOS cameras on XMM-Newton and the Proportional Counter Array (PCA) on RXTE. This represents the densest X-ray coverage of the source, although it must be said that the spectral measurements are significantly hampered by the high interstellar absorption (at energies below $2 \mathrm{keV}$ ) and source confusion (above $10 \mathrm{keV}$ ). The Gas Imaging Spectrometer (GIS) onboard ASCA also performed a long observation of SAX J1747.0-2853 in March 2000. Since this observation duplicates the coverage of the other instruments, both in time and instrumental capabilities, we choose not to analyze these data in great detail but merely checked the standard data products made available by NASA's High-Energy Astronomy Science Archive Research Center. Using all X-ray data, we studied the spectrum as a function of luminosity and tried to constrain accretion flow geometries at different states of the source. We start with a discussion of the long-term light curve in Sect. 2, continue with a treatment of the $0.3-10 \mathrm{keV}$ BeppoSAX-NFI spectrum during various stages of activity in Sect. 3, discuss the ASCA data in Sect. 4 and treat the XMM-Newton data in Sect. 5. In Sect. 6 we review the X-ray burst activity. We end with an interpretation of all data in Sect. 7.

\section{Long-term lightcurve and observations}

Since February 1999, the PCA instrument on board the RXTE satellite is programmed to scan the Galactic bulge twice a week with a sensitivity close to $1 \mathrm{mCrab}$ (Swank \& Markwardt 2001). Observations occur throughout the year, except for three

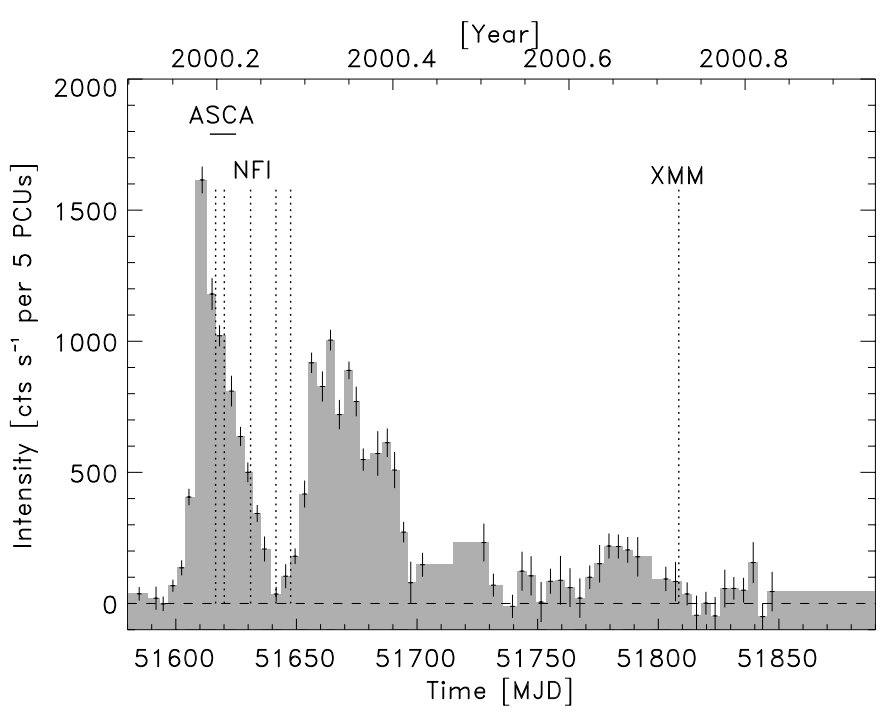

Fig. 2. The PCA lightcurve of the SAX J1747.0-2853 obtained between 2000 February 10 and October 30. The five BeppoSAX NFI observations and the later XMM-Newton observation are indicated.

months centered on December and one month in June, when solar constraints on spacecraft pointing prevent them. Figure 1 shows the lightcurve of SAX J1747.0-2853 resulting from these observations, from the beginning of the bulge scan program up to 2003 March 30. In source confused regions like this, it is very difficult to perform an a priori background subtraction. Rather we evaluated a background level a posteriori per each of two scan directions and subtracted that level from the data in the plots presented here. This level was verified from independent measurements with BeppoSAX and XMM. The light curve is characterized by six peaks in March 1999, March 2000, April 2000, August 2000, August 2001 and September 2001. The 1999 outburst is reported for the first time. We furthermore know that there was also an outburst in March 1998 (Sidoli et al. 1998; Natalucci et al. 2000).

The X-ray activity of SAX J1747.0-2853 appears strongest in 2000, with three peaks within 5 months of which the first one had the highest peak flux measured for the source thus far. SAX J1747.0-2853 was found to be in outburst on 2000 March 2 with an average intensity of $42 \mathrm{mCrab}$ in the $2-10 \mathrm{keV}$ band (Markwardt et al. 2000). The peak of $140 \mathrm{mCrab}$ occurred 6 days later. After that the brightness steadily decreased over the course of a month and reached a minimum at about 2000 April 7. Then the brightness increased again to a second peak of 80 mCrab on 2000 April 30. Subsequently, the source had another peak four months later in mid August at about $20 \mathrm{mCrab}$. Some outbursts may have been missed during observation interruptions. In conclusion, it appears SAX J1747.0-2853 exhibited outburst activity with a recurrence time of approximately one year between 1998 and 2001, with a peak flux of approximately one tenth that of the Crab and a duration between one week and a few months.

Figure 2 focuses on part of the lightcurve (in the year 2000) where the X-ray activity was the highest and the X-ray coverage the densest. Five BeppoSAX NFI observations were performed and one serendipitous XMM-Newton observation. 
The first four NFI observations are covering the decreasing part of the strongest peak, ending with an observation at the minimum between the two peaks (on April 7). The April 12 observation covers the rising phase of the 2 nd peak. The XMMNewton observation covers the tail of the third $20 \mathrm{mCrab}$ peaked outburst. Exact observation and exposure times are provided in Table 1.

The long ASCA observation was performed between March 11.1 and 22.0, 2000, with a net exposure time for the GIS of $331.0 \mathrm{ks}$. SAX J1747.0-2853 was outside the field of view of the Solid-state Imaging Spectrometer. The observation covers the first half of the decaying part of the brightest peak and overlaps the first two NFI observations.

It is worthwhile to note that the Chandra observation occurred a few weeks before the 2001 outburst. It is quite possible that this observation caught the source in the onset of that outburst, given that the sensitivity of the PCA observations for SAX J1747.0-2853 is a moderate 3 mCrab which is roughly equivalent to $5 \times 10^{35} \mathrm{erg} \mathrm{s}^{-1}$.

\section{BeppoSAX NFI observations and data analysis}

\subsection{Data extraction}

The NFIs on BeppoSAX (Boella et al. 1997a) consist of two imaging devices: the Low-Energy Concentrator Spectrometer (LECS; Parmar et al. 1997) operating in the 0.1-10 keV bandpass and the Medium-Energy Concentrator Spectrometer (MECS; Boella et al. 1997b) operating at 1.6-10 keV. The source was also observed by the non-imaging Phoswich Detector System (PDS; Frontera et al. 1997) operating in the $15-220 \mathrm{keV}$ bandpass, while the High-Pressure Gas Scintillation Proportional Counter was turned off. The circular fields of view of the LECS and MECS are 40 arcmin and 30 arcmin in diameter. The PDS has a hexagonal field of view with a diameter ranging between 1.3 and 1.5 (full width at half maximum FWHM). The energy resolution at $6 \mathrm{keV}$ for the MECS is $8 \%$ (FWHM).

To study the source spectrum we extracted the LECS and MECS data from circular regions centered at the source using radii of 4.6 arcmin and 4 arcmin and rebinned them in order to have at least 20 counts per photon energy bin, so that we could apply the $\chi^{2}$ statistic in fitting models to the data.

The photon flux as measured by the MECS remains constant within $10 \%$ for all observations except for the 2nd observation (on March 16) during which it steadily decreases by $40 \%$ and for the April 12 observation which shows an increase of $50 \%$ (note that this observation lasted $1.6 \mathrm{~d}$ ).

The time intervals corresponding to two X-ray bursts were excluded from the MECS data (see Sect. 6). The LECS was not observing during the bursts. We found that the standard LECS and MECS background spectra obtained from blank field observations underestimate the background present in empty regions close to the source by a factor of $\sim 5$. We therefore used background spectra for the LECS and MECS instruments obtained from a region near to the source and void of point sources. Since the source was very bright during the first two observations, the tails of the point-spread

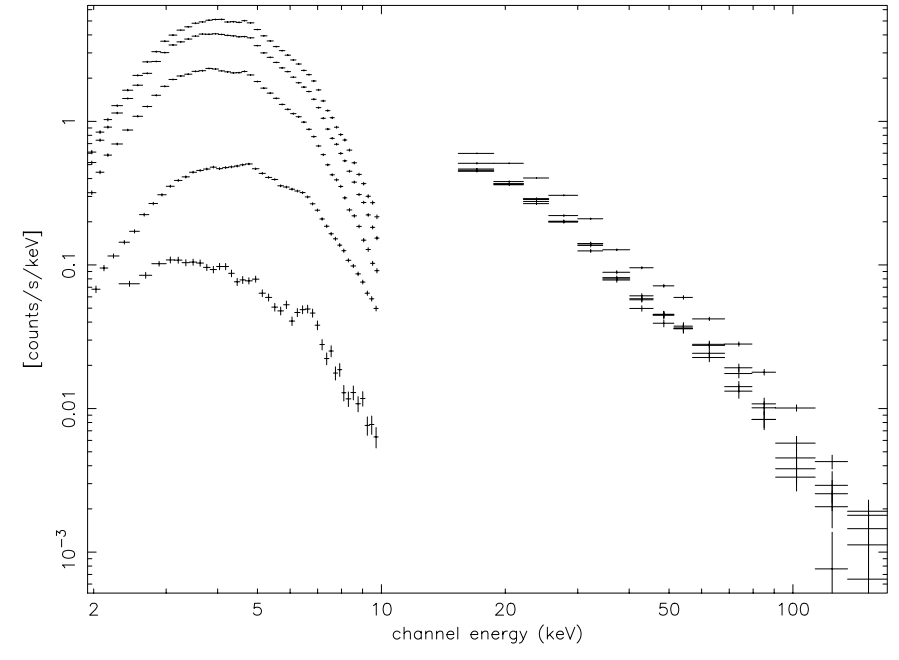

Fig. 3. MECS (1.8-10 keV) and PDS (15-200 keV) spectra of SAX J1747.0-2853 from 2000 March 13 (1st from the top), March 16 (2nd from the top), March 27 (3rd from the top) April 7 (1st from the bottom) and April 12 (2nd from the bottom). The MECS spectra are illustrating well the changes of the brightness of the source and the changes of the shape of the spectra. The small changes in the PDS spectra are indicative of contamination by other sources. Only the PDS data from the first observation are somewhat above the noise level created by other sources in the field of view of the PDS.

function became too bright and the local background determination became questionable. Therefore, the background subtraction for the MECS and LECS instrument was performed using backgrounds from the longest observation on April 12 . For the other three observations the background spectra and source spectra were extracted from the same image. Due to calibration uncertainties the bandpasses for the spectral analysis were limited to $0.3-4 \mathrm{keV}$ for the LECS, $1.8-10 \mathrm{keV}$ for the MECS and 15-200 keV for the PDS. In order to accommodate cross calibration uncertainties, the normalisations of the LECS and PDS data sets with respect to the MECS data were left as free parameters. Furthermore, a $1 \%$ systematic error per channel due to calibration uncertainties of the instruments is included. We fitted our spectra using a variety of models in XSPEC (version 11.2.0; Arnaud 1996).

By fitting the spectra with various models we found that the normalisation constant for the PDS data is unacceptably high (larger than 1.5). Since the PDS has a relatively large field of view and SAX J1747.0-2853 is in the central region of our Galaxy where the density of X-ray sources is high (cf., Sidoli et al. 1999), the high normalisation constant indicates that the PDS data are significantly contaminated by other sources. This is further illustrated by the small variability of the PDS spectrum as compared by the (uncontaminated) MECS spectrum (Fig. 3). Also, the contaminating sources (most notably $1 \mathrm{E} 1740.7-2942$ which is at a $64^{\prime}$ off-axis angle) are known to be strongly variable which precludes the usefulness of other PDS observations when SAX J1747.0-2853 was off as background measurements. We decided to exclude the PDS data from the analysis. 
Table 1. Results of modeling the BeppoSAX-NFI and XMM-Newton spectra (last entry) with a combination of a disk black body model (Mitsuda et al. 1984; Makishima et al. 1986), Comptonization of soft photons in a hot plasma (Titarchuk 1991), and a narrow emission line.

\begin{tabular}{|c|c|c|c|c|c|}
\hline $\begin{array}{l}\text { Observation date in } \\
2000\end{array}$ & Exposure & Model Parameters* & $\begin{array}{l}2-10 \text { and } \\
\text { keV unabs. }\end{array}$ & $\begin{array}{l}3-10 \\
x^{\dagger}\end{array}$ & $\chi_{v}^{2}$ (d.o.f.) \\
\hline Mar. 13.19-13.85 & $21.4 \mathrm{ks}$ & $\begin{array}{l}N_{\mathrm{H}}=8.8_{-0.3}^{+0.6} \\
k T_{\text {in }}=1.9 \pm 0.1, R_{\text {in }}^{2} \cos \theta=16.5_{-1.0}^{+2.5} \\
k T_{0}=0.48_{-0.20}^{+0.03}, k T_{\mathrm{e}}=\text { unconstrained, } \tau<3 \\
E_{\text {line }}=6.82 \pm 0.06, E W=35 \mathrm{eV}, f=(9.2 \pm 2.1)\end{array}$ & $\begin{array}{l}3.9 \times 10^{-9} \\
5.9 \times 10^{-9}\end{array}$ & (79) & $0.96(70)$ \\
\hline Mar. 16.71-17.48 & $34.4 \mathrm{ks}$ & $\begin{array}{l}N_{\mathrm{H}}=9.3_{-0.3}^{+0.7} \\
k T_{\text {in }}=1.83 \pm 0.02, R_{\mathrm{in}}^{2} \cos \theta=14.4_{-0.9}^{+1.8} \\
k T_{0}=0.4 \pm 0.1, k T_{\mathrm{e}}=\text { unconstrained, } \tau<0.2 \\
E_{\text {line }}=6.78_{-0.05}^{+0.07}, E W=41 \mathrm{eV}, f=8.6_{-2.1}^{+0.6}\end{array}$ & $\begin{array}{l}3.0 \times 10^{-9} \\
4.6 \times 10^{-9}\end{array}$ & $(82)$ & $1.09(73)$ \\
\hline Mar. 27.63-28.45 & $36.6 \mathrm{ks}$ & $\begin{array}{l}N_{\mathrm{H}}=8.8^{\mathrm{Il}} \\
k T_{\text {in }}=1.87_{-0.07}^{+0.02}, R_{\text {in }}^{2} \cos \theta=7.3_{-0.6}^{+5.5} \\
k T_{0}=0.41_{-0.05}^{+0.04}, k T_{\mathrm{e}}=\text { unconstrained, } \tau=\text { unconstrained } \\
E_{\text {line }}=6.53_{-0.07}^{+0.08}, E W=33.2 \mathrm{eV}, f=4.1_{-1.0}^{+1.1}\end{array}$ & $\begin{array}{l}1.7 \times 10^{-9} \\
2.6 \times 10^{-9}\end{array}$ & $(80)$ & $1.04(70)$ \\
\hline Apr. 7.09-7.82 & $27.9 \mathrm{ks}$ & $\begin{array}{l}N_{\mathrm{H}}=8.8^{\mathrm{q}} \\
k T_{\text {in }}=0.43 \pm 0.04, R_{\text {in }}^{2} \cos \theta=340_{-160}^{+280} \\
k T_{0}=0.16_{-0.01}^{+0.02}, k T_{\mathrm{e}}=\text { unconstrained, } \tau=\text { unconstrained } \\
E_{\text {line }}=6.75_{-0.06}^{+0.03}, E W=285 \mathrm{eV}, f=1.31_{-0.17}^{+0.19}\end{array}$ & $\begin{array}{l}9.3 \times 10^{-11} \\
4.3 \times 10^{-10}\end{array}$ & (17) & $1.00(55)$ \\
\hline Apr. $12.82-14.40$ & $57.2 \mathrm{ks}$ & $\begin{array}{l}N_{\mathrm{H}}=8.8^{\mathbb{I}} \\
k T_{\text {in }}=2.9 \pm 0.3, R_{\text {in }}^{2} \cos \theta=0.20 \pm 0.04 \\
k T_{0}<1.3, k T_{\mathrm{e}}=\text { unconstrained, } \tau=\text { unconstrained } \\
E_{\text {line }}=6.56_{-0.03}^{+0.09}, E W=58.5 \mathrm{eV}, f=2.3_{-0.3}^{+0.4}\end{array}$ & $\begin{array}{l}4.0 \times 10^{-10} \\
7.1 \times 10^{-10}\end{array}$ & $(58)$ & $1.02(64)$ \\
\hline Sept. 21.39-21.63 & $\begin{array}{l}\text { MOS } \\
16.7 \mathrm{ks}, \\
\text { EPIC-pn } \\
7.6 \mathrm{ks}\end{array}$ & $\begin{array}{l}N_{\mathrm{H}}=8.8^{\mathrm{I}} \\
k T_{\mathrm{in}}=0.3 \pm 0.1, R_{\mathrm{in}}^{2} \cos \theta=7.0_{-0.6}^{+59} \times 10^{2} \\
k T_{0}=0.6 \pm 0.1, k T_{\mathrm{e}}=39_{-7}^{+128}, \tau=0.15_{-0.10}^{+0.42}\end{array}$ & $\begin{array}{l}8.7 \times 10^{-11} \\
6.6 \times 10^{-10}\end{array}$ & (1) & 1.38 (139) \\
\hline
\end{tabular}

\footnotetext{
Net exposure time as it applies to the MECS data;
}

* Parameters and units: $N_{\mathrm{H}}$ : hydrogen column density in units of $10^{22} \mathrm{~cm}^{-2} ; k T_{\mathrm{in}}$ : temperature at inner disk radius in keV; $R_{\mathrm{in}}^{2} \cos \theta: \theta$ is the disk inclination angle, $R_{\mathrm{in}}$ is the disk inner radius in kilometers for a source at $10 \mathrm{kpc} ; k T_{0}$ : temperature of the Comptonized soft seed photons in $\mathrm{keV} ; k T_{\mathrm{e}}$ : temperature of the electron plasma in $\mathrm{keV} ; \tau$ : optical depth of the plasma; $E_{\text {line }}$ : centroid energy of Gaussian line profile in keV; $E W$ : equivalent width of the line; $f$ : line flux in $10^{-4}$ phot $\mathrm{s}^{-1} \mathrm{~cm}^{-2}$.

${ }^{\dagger}$ Unabsorbed flux in units of ergs $\mathrm{cm}^{-2} \mathrm{~s}^{-1}$. The typical uncertainty in this number is $10 \%$ for $2-10 \mathrm{keV}$ and a factor of 2 for $0.3-10 \mathrm{keV}$. The number between parentheses refers to the fraction in percents of detected photons coming from the blackbody component.

II The value was frozen.

\subsection{Spectral modeling}

We tested different spectral models to describe the continuum. To describe the soft component we tested the blackbody and the multicolor disk blackbody model, for the hard component we tested a powerlaw, an exponentially cut off powerlaw and a Comptonization model. A more elaborate discussion is presented in Natalucci et al. (2004). The best fitting model of the continuum consists of a soft multicolor disk blackbody component and a hard Comptonized component, all with interstellar absorption. This is in agreement with results obtained by Natalucci et al. (2000) for NFI data on the 1998 outburst of SAX J1747.0-2853, and with those on many other LMXBs at similar flux levels (e.g., Sidoli et al. 2001). However, we note that the combination of a disk black body and a power law fits just as well due to the absence of PDS data (in contrast to the BeppoSAX study of the 1998 outburst by Natalucci et al. 2000).
The above-mentioned continuum model describes the data reasonably. However, the reduced $\chi^{2}$ of the fit still exceeds 2 and by investigating the residuals we noticed that there is a narrow-band spectral component between 5.5 and $7.5 \mathrm{keV}$, which is not represented by these models. The peak of the narrow-band emission feature is near the energy expected for the Fe-K line complex. To describe this feature we included a Gaussian in our model. We found that a Gaussian with the width fixed to zero (a narrow line) describes the emission feature well. The $1 \sigma$ width of the line is with $90 \%$ confidence smaller than $0.28 \mathrm{keV}$. We note that the background in the vicinity of the source does not show any sign of an $\mathrm{Fe}-\mathrm{K}$ line. This is consistent with the expected flux from diffuse $\mathrm{Fe}-\mathrm{K}$ line in this region of at most $3 \times 10^{-5} \mathrm{ph} \mathrm{s}^{-1} \mathrm{~cm}^{-2}$ for a $4^{\prime}$ extraction radius (e.g., Koyama et al. 1986; Wang et al. 2002). In Table 1 we present the parameter values for each observation together with single-parameter $1 \sigma$ errors, for a model consisting of a soft component (disk blackbody), a hard Comptonized 
component and a Gaussian, all with interstellar absorption. We get lower values of $N_{\mathrm{H}}$ for the data obtained in the last three observations when the brightness of the source was lower. Since it is most likely that $N_{\mathrm{H}}$ is constant in the direction of the source (circumstellar $N_{\mathrm{H}}$ variations usually are accompanied with dipping activity in the light curve which is not detected), we fixed $N_{\mathrm{H}}$ to $8.8 \times 10^{22} \mathrm{~cm}^{-2}$. We decided to choose this value because it was determined from data with the highest count rate and highest accuracy.

It is evident from Fig. 3 and Table 1 that the spectrum is changing over time. The contribution of the disk black body flux to the total observed photon flux in $2-10 \mathrm{keV}$ is decreasing with the overall flux. The spectrum is also slightly hardening with time. If we replace the Comptonization model by a power law to quantify the changes in a straightforward manner, the power law photon index decreases from 2.64 on March 13 to 2.30 on April 12. The most significant decrease of the $0.3-10 \mathrm{keV}$ unabsorbed flux happened between March 28 and April 7. In this time interval there were significant changes in the disk blackbody component and in the equivalent width of the iron line. Between March 28 and April 7 the inner disk temperature decreased by a factor of $\sim 5$ and the disk radius increased. At the same time the equivalent width of the iron line increased by a factor of $\sim 7$. Between April 7 and April 12 the inner disk temperature jumped from $\sim 0.4 \mathrm{keV}$ to $\sim 3.1 \mathrm{keV}$, the disk radius decreased and the equivalent width of the iron line decreased by a factor of $\sim 5$. We do not find any significant changes in the parameter values of the Comptonized component, but note that the data are not particularly constraining due to the lack of data above $10 \mathrm{keV}$. The spectrum obtained during the observation on April 7 is shown in Fig. 4.

\section{ASCA observation}

SAX J1747.0-2853 decayed during the ASCA observation by $40 \%$. Furthermore, the light curve shows two X-ray bursts. The overall spectrum as measured with ASCA-GIS2 shows the same components as the NFI spectrum $\left(\chi_{\mathrm{r}}^{2}=1.22\right.$ for 669 d.o.f.) with an $\mathrm{Fe}-\mathrm{K}$ line at $6.70_{-0.02}^{+0.04} \mathrm{keV}$ with a flux of $(1.1 \pm 0.2) \times 10^{-3}$ phot s $\mathrm{s}^{-1} \mathrm{~cm}^{-2}$ and an equivalent width of $45 \mathrm{eV}$. These results confirm the findings of the first two NFI observations.

\section{XMM-Newton observation and data analysis}

There are three identical telescopes on board of the XMMNewton satellite. For two of these, $50 \%$ of the radiation is diverted to reflection gratings and the other $50 \%$ of the radiation is collected by the EPIC metal oxide semoconductor MOS1 and MOS2 CCD arrays. $100 \%$ of the radiation from the third telescope is collected by the EPIC-pn CCD array. The 3 EPIC cameras have $\sim 6^{\prime \prime}$ resolution in a $30^{\prime}$-diameter field of view. The energy resolution intrinsic to all CCDs is $E / \delta E=20$ to 50 in a 0.1 to $15 \mathrm{keV}$ bandpass.

During the observation on SAX J1747.0-2853 all cameras were operated in full-window mode with the medium filter. The data analysis was performed with the Science Analysis System (SAS, version 5.4.1). Several background flares occurred during the observation, relevant data were excluded from the analyzis. We did not use the data from time intervals during which the count rate of photons with energy higher then 10 $\mathrm{keV}$ exceeds 0.5 counts $\mathrm{s}^{-1}$ for MOS and 2 counts $\mathrm{s}^{-1}$ for the EPIC-pn. The resulting net exposure times are $7.6 \mathrm{ks}$ for the pn camera and $16.7 \mathrm{ks}$ for each of the MOS cameras. The source spectrum was extracted from a circular region centered at the source using a diameter of $1.5 \mathrm{arcmin}$. The background spectra were extracted from a circle with the same radius, from a region close to SAX J1747.0-2853 from the same CCD. The extracted spectra were rebinned into bins with a minimum of 20 counts per bin. The response matrices and ancillary response files were generated using SAS.

The bandpasses for the spectral analyses are, due to calibration uncertainties, limited for all three EPIC cameras to 0.3-10.0 keV. We fitted the obtained spectra using XSPEC. In order to accommodate the cross calibration uncertainties the normalisations for the MOS1 and MOS2 data sets with respect to the EPIC-pn data were left as free parameters. We started with the same continuum model as we used for the BeppoSAX observations. The spectrum with the best fit and residuals is shown in Fig. 5. There was no need to include an iron line feature in the model. Our data constrain the highest possible value for the iron line flux to $1.85 \times 10^{-5}$ phot s${ }^{-1} \mathrm{~cm}^{-2}$ with a $90 \%$ confidence level. This value is an order of magnitude lower then the lowest value detected by the BeppoSAX observations after the outburst. The upper limit of the equivalent width of the iron line is $24.7 \mathrm{eV}$. Taken literally, the temperature at the inner disk radius was small and the disk was far away from the neutron star compared to the previous observations. The unabsorbed $2-10 \mathrm{keV}$ flux during this observation was $8.7 \times 10^{-11} \mathrm{erg} \mathrm{cm}^{-2} \mathrm{~s}^{-1}$. This value is comparable to the April 7 BeppoSAX observation. The data obtained by the EPIC-pn show some excess with respect to the model at low energies, but the data obtained by the MOS cameras do not confirm the excess.

\section{X-ray bursts}

Two type-I X-ray bursts were detected with the MECS. The first burst started on 2000 March 17 at 0:14:30 UT and lasted approximately $20 \mathrm{~s}$. Its time profile has a fast rise and an exponential decay. We fitted the decaying part with an exponential and find an e-folding decay time of $6.6 \pm 0.4 \mathrm{~s}$. The second burst started on 2000 April 12 at 23:14:23 UT and lasted $50 \mathrm{~s}$. It took about $15 \mathrm{~s}$ for the decay to set in for this burst while for the previous burst the decay set in after about $2 \mathrm{~s}$. The e-folding decay time is $8.0 \pm 0.3 \mathrm{~s}$. The neutron star photospheric radius expansion as well as a plateau in the peak flux during the bursts suggests that the bursts are Eddington-limited type I X-ray bursts. When fitted with a blackbody spectrum, the burst peak intensity corresponds to an unabsorbed bolometric flux of $(4.47 \pm 1.4) \times 10^{-8} \mathrm{ergs} \mathrm{s}^{-2}$. Assuming isotropic burst emission, Eddington limited type I X-ray bursts can be used to constrain the distance of the source. Using a standard candle luminosity of $(3.0 \pm 0.6) \times 10^{38} \mathrm{ergs} \mathrm{s}^{-1}$ (Lewin et al. 1993; see also Kuulkers et al. 2003), we calculated a distance of $7.48 \pm 1.34 \mathrm{kpc}$ for SAX J1747.0-2853. 


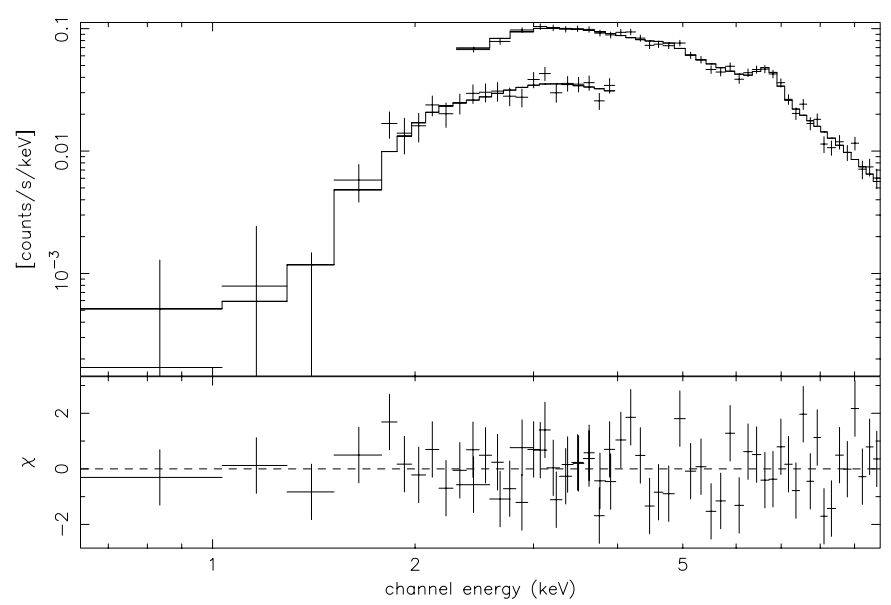

Fig. 4. Spectrum of the SAX J1747.0-2853 obtained during the observation on 2000 April 7, when the source was in the minimum between the two peaks. The spectrum is fitted with Comptonization, disk blackbody and a narrow line, all with absorption. The equivalent width of the iron line at this observation is the highest of all observations.

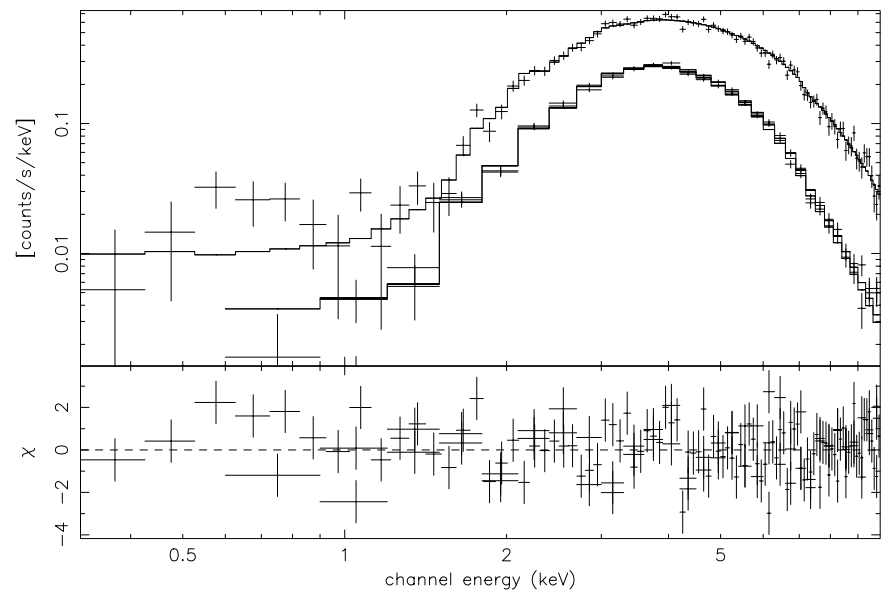

Fig. 5. Spectrum of the SAX J1747.0-2853 obtained by the XMMNewton EPIC cameras during the observation on 2000 September 21, with the best fit and $1 \sigma$ residuals. The spectrum is fitted as the sum of a disk blackbody and a Comptonized component all with absorption. The upper curve shows the data obtained by the EPIC-pn, the lower curves show the MOS1 and MOS2 data.

We investigated the burst history of the SAX J1747.0-2853 by employing the WFCs (for an instrument description, see Jager et al. 1997). The PCA lightcurve is overplotted in Fig. 6 with the time intervals of the WFC observations and the times of detected bursts. There were 30 bursts detected during or close to outbursts in this period. It is interesting to note that the BeppoSAX WFCs had SAX J1747.0-2853 in the field of view during the whole first peak of the outburst, but bursts were detected only at or after peak flux. The chance probability of detecting zero bursts prior to the peak, assuming that the average burst rate is identical to that after the peak and that the bursts arrive randomly, is $4.6 \%$.

The WFCs did not detect any bursts during its fall 1999 (MJD 51410-51470) campaign on the Galactic center when there was no strong persistent X-ray activity, while the net exposure time of this campaign was $801 \mathrm{ks}$. At other times when

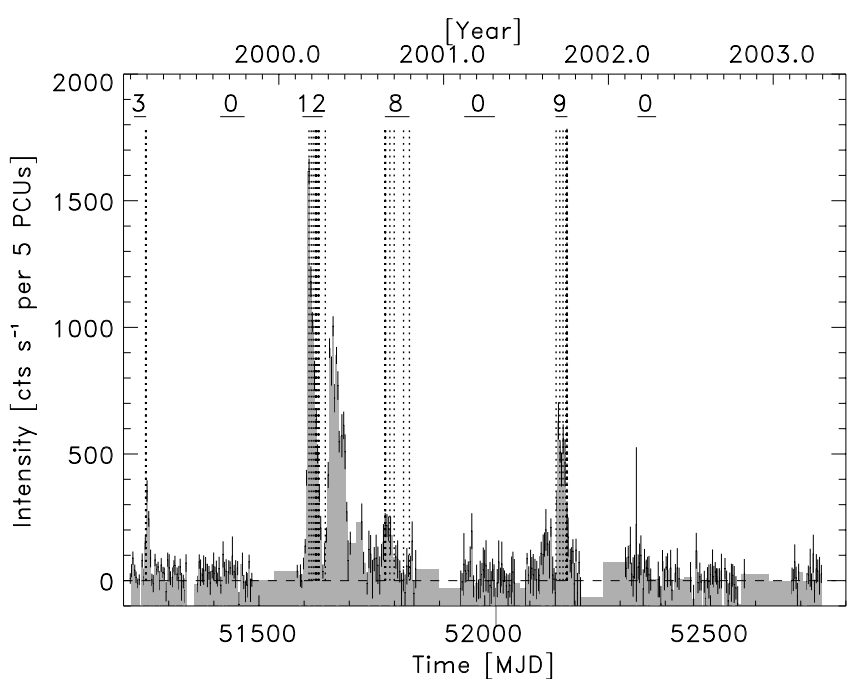

Fig. 6. The PCA lightcurve of SAX J1747.0-2853 with the times (as indicated by horizontal lines) when the BeppoSAX WFC had the source in its field of view with sufficient sensitivity, and with the times of the detected X-ray bursts (as indicated by vertical dotted lines; this includes the four NFI and ASCA detected bursts). The number of the detected X-ray bursts in an observation window is indicated over the horizontal lines.

Table 2. Summary of the twelve Galactic center observation campaigns of the WFCs. Shown are the time frames of the campaigns, the number of observations, the net exposure time and the number of bursts seen from SAX J1747.0-2853.

\begin{tabular}{lcrrr}
\hline \hline & Campaign & \# obs. & $t_{\text {exp }}(\mathrm{ks})$ & \# bursts \\
\hline 1996 & Aug. 15-Oct. 29 & 67 & 1017 & 0 \\
1997 & Mar. 02-Apr. 26 & 21 & 654 & 0 \\
1997 & Sep. 06-Oct. 12 & 13 & 302 & 0 \\
1998 & Feb. 11-Apr. 11 & 17 & 551 & 14 \\
1998 & Aug. 22-Oct. 23 & 10 & 410 & 0 \\
1999 & Feb. 14-Apr. 11 & 14 & 470 & 3 \\
1999 & Aug. 24-Oct. 17 & 24 & 801 & 0 \\
2000 & Feb. 18-Apr. 07 & 21 & 633 & 8 \\
2000 & Aug. 22-Oct. 16 & 29 & 767 & 8 \\
2001 & Feb. 14-Apr. 23 & 5 & 215 & 0 \\
2001 & Sep. 04-Sep. 30 & 7 & 284 & 9 \\
2002 & Mar. 05-Apr. 15 & 5 & 91 & 0 \\
\hline
\end{tabular}

the PCA lightcurve also does not show strong persistent activity, there are considerable amounts of bursts (for instance in the period around MJD 51800). This strongly suggests that the source was truly in quiescence during the fall of 1999. The presence of truly quiescent periods is furthermore supported if we consider the full WFC database of 42 burst detections from SAX J1747.0-2853, see Table 2. There are many prolonged periods when there were no bursts detected, the coverage was particularly extensive in the fall of 1996 and the spring of 1997 with a combined exposure time of $1.7 \mathrm{Ms}$. Finally, we note that the Chandra observation as discussed by Wijnands et al. (2002) was close to the 2001 outburst during which WFC also detected 9 bursts and the above-quiescent flux very likely reflects the onset of that outburst. 


\section{Discussion}

Whenever its location was covered by $2-10 \mathrm{keV}$ instrumentation with $\sim 10^{-12} \mathrm{erg} \mathrm{cm}^{-2} \mathrm{~s}^{-1}$ sensitivity or better (we exclude soft X-rays here because the low-energy absorption is very high), SAX J1747.0-2853 was detected to be above quiescence. The lowest reported flux is $1.9 \times 10^{-11} \mathrm{erg} \mathrm{cm}^{-2} \mathrm{~s}^{-1}(2-10 \mathrm{keV})$ during a Chandra observation in July 2001 (Wijnands et al. 2002). The next lowest flux value was measured by Sidoli et al. (1998) at $5 \times 10^{-11} \mathrm{erg} \mathrm{cm}^{-2} \mathrm{~s}^{-1}$ in April 1998 in the tail of the 1998 outburst. For a distance of $7.5 \mathrm{kpc}$ this translates to 2 and $4 \times 10^{35} \mathrm{erg} \mathrm{s}^{-1}$. An X-ray burst was detected during the observation that Sidoli et al. discuss, independently testifying to low-level accretion activity. Based on the failure to find SAX J1747.0-2853 in true quiescence, it appears that SAX J1747.0-2853 is not a true transient. This suggestion was recently reaffirmed by the report of an X-ray burst from SAX J1747.0-2853 in 1991 (Grebenev et al. 2002) which is seven years before the source discovery. However, we find indirect evidence that it does go to quiescence between outbursts. The lack of burst detections in 3 WFC campaigns, each with a coverage in excess of $600 \mathrm{ks}$, is that evidence. If there were any significant accretion, there would have been bursts as shown by Sidoli et al. (1999).

From the long-term PCA lightcurve we estimate the average $0.3-10 \mathrm{keV}$ luminosity. The average count rate is $87 \pm$ $2 \mathrm{c} \mathrm{s}^{-1}$ per 5 PCUs. This is within 5\% from the value measured within a day from the XMM-Newton observation. Applying the results of that observation we estimate an average flux of $(6 \pm$ $3) \times 10^{-10} \mathrm{erg} \mathrm{cm}^{-2} \mathrm{~s}^{-1}$ or luminosity of $(4 \pm 2) \times 10^{36} \mathrm{erg} \mathrm{s}^{-1}$. The bolometric luminosity is estimated to be at most $50 \%$ more. Thus, the system appears to be close to the critical luminosity below which the accretion onto the compact object changes from persistent to transient in nature (van Paradijs 1996; White et al. 1984).

The multiple-peak outburst of 2000 is an interesting feature, as is the otherwise somewhat irregular outburst behavior. The suggestion presents itself that the accretion disk is not fully emptied during mosts outbursts due to heating fronts in the disk that do not make it to either edge of the disk (see Lasota 2001 and references therein). The fact that during the rise to the first peak no X-ray bursts were detected while they were during the decay suggests that this outburst was caused by an outside-in heating front in the accretion disk. If it would have been an inside-out front, one would have expected immediate accretion onto the neutron star surface and, therefore, ignition of X-ray bursts fairly soon after the onset of the outburst.

Our series of five NFI observations nicely probes the outburst evolution, with coverage of a decaying state of the first peak and the rising state of the second peak. The most conspicuous changes in the spectrum during the decay are the softening of the soft component (as diagnosed by the disk inner edge temperature) and the jump of the equivalent width of the $\mathrm{Fe}-\mathrm{K}$ line. This change is most obvious in the last NFI observation of the decay with the lowest flux (see Fig. 3) for which the MECS spectrum shows an upturn at low energies (when $k T_{\text {in }}$ decreases from roughly 2 to $0.4 \mathrm{keV}$ ) and a larger $\mathrm{Fe}-\mathrm{K}$ line (the equivalent width increases from about $40 \mathrm{eV}$ to about $300 \mathrm{eV}$ ). Also, the temperature of the seed photons for the Comptonization drops threefold in this observation. The temperature measurements are qualitatively consistent with a picture of a changing inner radius of the optically thick part of the accretion disk (e.g., review by Lasota 2001). When the flux becomes lower during the first peak, the optically thick part of the disk moves away from the neutron star and the disk spectrum cools. The rise to the second outburst peak was covered by the last NFI observation. The spectrum is characterized by a relatively hot disk temperature, hotter than any previous measurement. Perhaps the optically thick part of the accretion disk here is closer to the neutron star than during the decay of the previous peak. Perhaps this reflects a disk instability that occurred in the inner parts of the disk instead and the outburst is the result of an inside-out disk instability. Unfortunately, we were not able to verify this with WFC monitoring observations of X-ray bursts.

There is one striking measurement regarding the $\mathrm{Fe}-\mathrm{K}$ line: the equivalent width right between the two outburst peaks is an order of magnitude larger than at other times and the line energy remains at a highly ionized level. The width is at least 10 times smaller during the XMM-Newton measurement which is at approximately the same persistent flux level (this is nicely illustrated in Figs. 4 and 5). We suggest that this is due to the fact that the hard X-ray continuum flux (i.e., photons more energetic than $\sim 10 \mathrm{keV}$ ), which must be responsible for the fluorescence, has not decreased by the same amount as the soft $\mathrm{X}$-ray flux, and that this flux is capable of ionizing the medium containing the iron, suggesting that the iron is not in the accretion disk but close to the source of the high-energy photons. A similar trend of the $\mathrm{Fe}-\mathrm{K}$ line energy and equivalent width has been seen in another transient and black hole candidate (GX 339-4) by Feng et al. (2003). They suggest that this may be explained by the presence of an advection-dominated accretion flow (e.g., Narayan \& Yi 1994). The results obtained by Wang et al. (2002) with Chandra on the hundreds of faint X-ray point sources in the center square degree of the Galaxy suggest that many near-to quiescent $\mathrm{X}$-ray binaries may have 6.7 keV Fe-K emission.

Acknowledgements. N.W. thanks the European Community for financial support. Four of the five BeppoSAX NFI observations were obtained from the publicly available archive provided by the Science Data Center of the Italian Space Agency (ASI). We thank the Data Center for pre-processing the data. We thank T. Oosterbroek for providing us a LECS response matrix for an uncommon accumulation radius. BeppoSAX was a joint Italian and Dutch program.

\section{References}

Arnaud, K. 1996, Astronomical Data Analysis Software and Systems, ed. G. Jacoby \& J. Barnes (San Francisco: ASP), ASP Conf. Ser., 101, 17

Boella, G., Butler, R. C., Perola, G. C., et al. 1997a, A\&AS, 122, 299

Boella, G., Chiappetti, L., Conti, G., et al. 1997b, A\&AS, 122, 327

Eisenhauer, F., Schödel, R., \& Genzel, R. 2003, ApJ, 597, L121

Feng, Y. X., Zhang, S. N., Sun, X., et al. 2001, ApJ, 553, 394 
Frontera, F., Costa, E., Dal Fiume, D., et al. 1997, A\&AS, 122, 357

Grebenev, S. A., Lutovinov, A. A., Pavlinsky, M. N., \& Sunyaev, R. A. 2002, Astron. Lett., 28, 799

In't Zand, J. J. M., Bazzano, A., Cocchi, M., et al. 1998, IAUC, 6846

Jager, R., Mels, W. A., Brinkman, A. C., et al. 1997, A\&AS, 125, 557

Koyama, K., Makishima, K., \& Tanaka, Y. 1986, PASJ, 38, 121

Kuulkers, E., Hartog, P. R., In't Zand, J. J. M., et al. 2003, A\&A, 399, 663

Lasota, J.-P. 2001, NewAR, 45, 449

Lewin, W. H. G., van Paradijs, J., \& Taam, R. E. 1993, Space Sci. Rev., 62, 223

Makishima, K., Maejima, Y., Mitsuda, K., et al. 1986, ApJ, 308, 635

Markwardt, C. B., Marshall, F. E., \& Swank, J. 2000, IAUC, 7377

Mitsuda, K., Inoue, H., Koyama, K. et al. 1984, PASJ, 36, 741

Natalucci, L., Bazzano, A., Cocchi, M., et al. 2000, ApJ, 543, L73

Natalucci, L., Bazzana, A., Cocchi, M., et al. 2004, A\&A, in press

Parmar, A. N., Martins, D. D. E., Bavdaz, M., et al. 1997, A\&AS, 122, 309
Proctor, R. J., Skinner, G. K., \& Willmore, A. P. 1978, MNRAS, 185, 745

Sidoli, L., Mereghetti, S., Israel, G. L., et al. 1998, A\&A, 336, L81

Sidoli, L., Mereghetti, S., Israel, G. L., et al. 1999, ApJ, 525, 215

Sidoli, L., Parmar, A. N., Oosterbroek, T., et al. 2001, A\&A, 368, 451 D. Dal Fiume

Strohmayer, T. E., \& Bildsten, L. 2003, in Compact Stellar X-Ray Sources, ed. W. H. G. Lewin, \& M. van der Klis (Cambridge University Press), in press [astro-ph/0301544]

Swank, J., \& Markwardt, C. 2001, Proc. New Century of X-ray Astronomy, ed. H. Inoue \& H. Kunieda, PASP Conf. Ser., 94

Titarchuk, L. 1994, ApJ, 434, 570

van Paradijs, J. 1996, ApJ, 464, L139

Wang, Q. D., Gotthelf, E. V., \& Lang, C. C. 2002, Nature, 415, 148

White, N. E., Swank, J. H., \& Kaluzienski, J. L. 1984, in High Energy Transients in Astrophysics, ed. S. Woosley, AIP, 31

Wijnands, R., Miller, J. M., \& Wang, D. Q. 2002, ApJ, 579, 422 